\title{
Entre plaisir et censure, Marie Taglioni chorégraphe du Second Empire
}

Between pleasure and censure: Marie Taglioni Choreographer of the Second

French Empire

\section{Vannina Olivesi}

\section{(2) OpenEdition}

\section{Journals}

Édition électronique

URL : https://journals.openedition.org/clio/13699

DOI : 10.4000/clio.13699

ISSN : $1777-5299$

\section{Éditeur}

Belin

Édition imprimée

Date de publication : 1 décembre 2017

Pagination : 43-64

ISBN : 978-2-410-00859-3

ISSN : 1252-7017

\section{Référence électronique}

Vannina Olivesi, «Entre plaisir et censure, Marie Taglioni chorégraphe du Second Empire », Clio.

Femmes, Genre, Histoire [En ligne], 46 | 2017, mis en ligne le 01 décembre 2020, consulté le 29 avril 2022. URL : http://journals.openedition.org/clio/13699; DOI : https://doi.org/10.4000/clio.13699

Tous droits réservés 


\section{Entre plaisir et censure, Marie Taglioni chorégraphe du Second Empire}

Née à Stockholm en 1804, Marie Taglioni est la fille du danseur et maitre de ballet italien Filippo Taglioni (1777-1871), fils de maître, et de Sophie Hedvig Karsten (1783-1862), harpiste professionnelle issue d'une famille suédoise d'acteurs dramatiques et lyriques renommés. Marie se forme à la danse théâtrale entre 1815 et 1822 et réalise ses débuts à la scène au Kärntnertortheater de Vienne avant d'être engagée à l'Opéra en 1827 où elle renouvelle bientôt l'art de la danse théâtrale en imposant une nouvelle esthétique. Sa maitrise de la technique des pointes et de l'arabesque la consacre rapidement, grâce à son interprétation de La Sylphide ${ }^{1}$, comme la plus grande ballerine du répertoire romantique. Après s'être produite dans les théâtres des principales capitales culturelles européennes, elle revient en 1858 négocier ses talents de pédagogue et de chorégraphe auprès de la direction de l'Opéra. Elle est d'abord nommée à un emploi d'Inspectrice des classes de danse en 1859, puis dirige la classe de perfectionnement à l'Opéra jusqu'en 1870. Durant cette période, elle compose les chorégraphies de deux ballets : Le Papillon (1860), qui remporte un franc succès, puis Zara ${ }^{2}$ dont la programmation en 1863 est empêchée par un accident survenu à Emma Livry, son interprète principale.

1 La Sylphide, ballet de Filippo Taglioni, livret d'Adolphe Nourrit, partition de JeanMadeleine Schneitzhoeffer, création le 12 mars 1832.

2 Les répétitions, commencées le 28 octobre 1862, cessent le 16 mars 1863. 


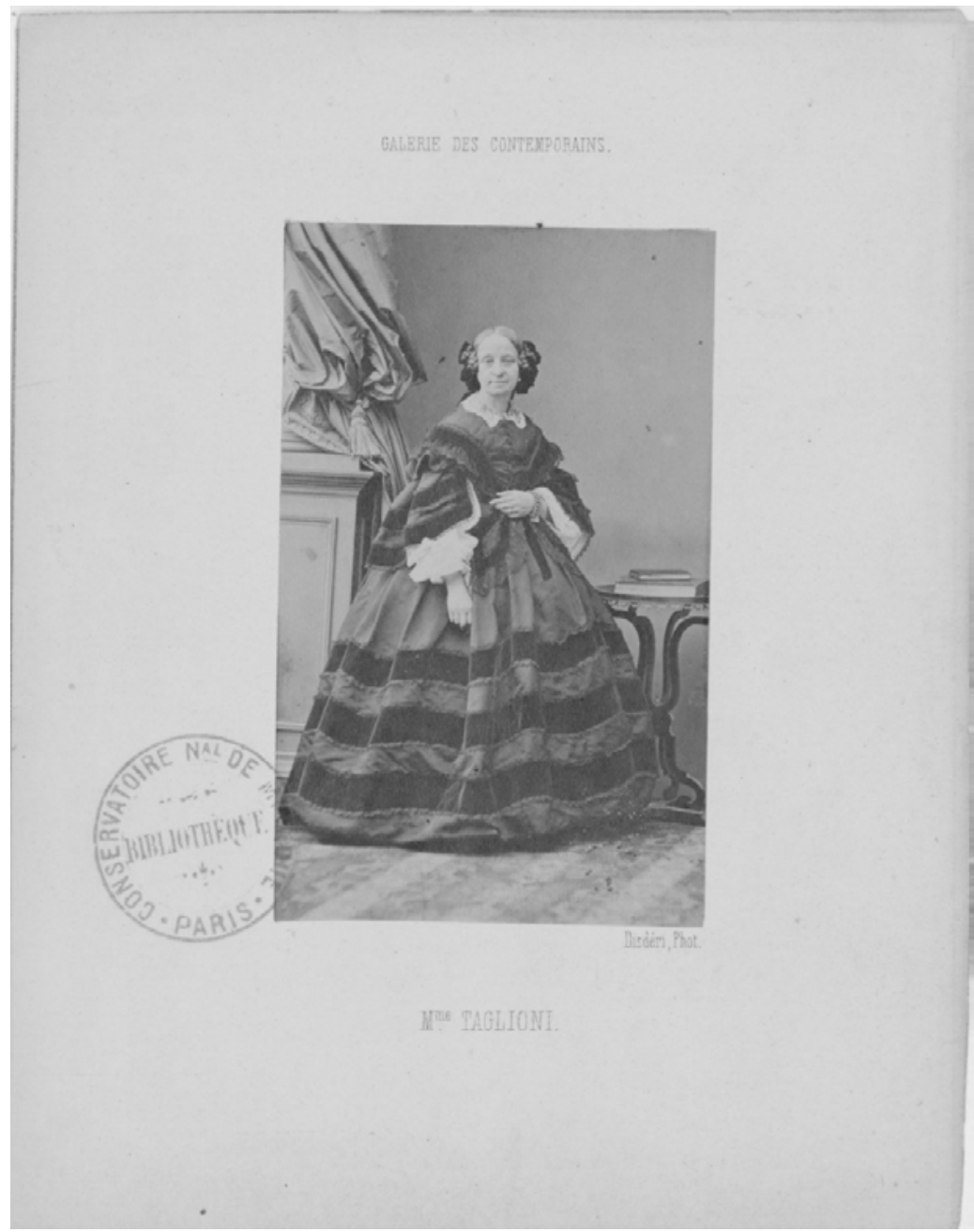

Fig. 1. Mme Taglioni, Extrait de la série « Galerie des Contemporains » (photographe Disdéri, 1862) (C BnF, Gallica. Pour une brève analyse, se reporter à la figure en fin d'article. 
Après Thérèse Elssler et Fanny Cerrito qui ont respectivement œuvré aux chorégraphies de La Volière en 1838 et Gemma en 1854², Marie Taglioni est la troisième ballerine parvenue à s'imposer chorégraphe dans une entreprise de spectacle organisée depuis sa fondation en 1669 sur une division sexuée du travail assignant les femmes à l'interprétation tandis que seule l'élite des danseurs de l'Opéra ou des théâtres étrangers accède aux emplois de maitre de ballet ${ }^{4}$. Cette exclusion des femmes des emplois dédiés à la composition se perpétue avec d'autant plus d'efficacité qu'elle n'est jamais explicitement énoncée dans les sources normatives définissant le mode de recrutement du personnel. Les danseuses exercent leurs compétences en qualité de chorégraphe, c'est-à-dire libéralement et sous le régime du droit d'auteur, à une époque où les maitres de ballet cumulent leur statut d'auteur de chorégraphies avec celui, plus protecteur, de maitre de ballet salarié par l'entreprise de spectacle. Le cas de Marie Taglioni est intéressant parce que la richesse des sources qui documentent sa carrière - correspondance professionnelle, documents administratifs, écrits du for privé, iconographie - offre l'occasion de replacer sa trajectoire professionnelle dans un contexte de forte féminisation du secteur de la danse théâtrale parisienne. Ses Sowvenirr ${ }^{5}$ inachevés constituent en outre une porte d'entrée précieuse pour saisir ses modes de pensée, retracer le rapport complexe qu'elle entretient avec l'acte de composer. Car les sources donnent à lire - à déceler au creux des silences - une Marie Taglioni consciente des enjeux moraux, économiques, sociaux et professionnels qu'implique sa reconversion.

Elle est dans sa cinquante-quatrième année lorsqu'elle réalise à l'automne 1858 le projet de réintégrer l'Opéra de Paris et, en cela, elle se distingue d'Elssler et Cerrito qui assumèrent publiquement dès le

3 La Volière ou les oiseaux de Bocacce, ballet de Thérèse Elssler, livret d'Eugène Scribe, musique de Casimir Gide, création le 5 mai 1838; Gemma, ballet de Fanny Cerrito, livret de Théophile Gautier, partition de Nicolò Gabrielli, création le 31 mai 1854.

4 Garafola 2005 ; McCleave $2007^{\mathrm{a}}$ et 2007b ; Marquié 2008 : 107-124 ; 2013 : 7588 ; 2015 ; 2016 : 202-206. Gustche-Miller 2015 : 56, 69-76.

5 Taglioni 2017. La chronologie de la rédaction demeure incertaine ; Bruno Ligore la situe autour de 1876 . 
début de leur carrière d'interprète des premiers essais dans la mise en scène et la composition ${ }^{6}$. Or, Taglioni relate accorder depuis sa jeunesse un intérêt sensible, bien que dissimulé, à la composition chorégraphique. Dans ses Somvenirs elle relie son initiation à l'improvisation et à la composition à la pratique de la danse dans l'espace privé aux côtés de sa mère ou en contexte de sociabilité, puis sur scène à l'occasion de ses débuts viennois. Elle trouve également auprès de son père les ressources nécessaires - réseau professionnel, formation technique, soutien bienveillant-pour développer ses talents dans la transmission et la composition de son art. Comme dans le cas des musiciennes, la famille joue un rôle crucial dans le développement de son autonomie artistique ${ }^{7}$.

\section{Transmission maternelle}

Entre 1814 et 1819, les époux Taglioni sont souvent séparés, Filippo réalisant de nombreuses tournées en Europe tandis que Sophie s'installe seule à Paris avec Marie et ses fils, Gustave et Paul. Marie intègre à cette époque le cours privé du pédagogue Jean-François Coulon, danseur et pédagogue français réputé 8 . Devenue adulte, Marie ne tarit pas d'éloges dans ses Souvenirs pour celle qu'elle présente telle une «excellente musicienne» ${ }^{9}$ avec «un talent remarquable $»^{10}$ dans la pratique de la harpe. Sophie Taglioni, qui a exercé son art en qualité d'instrumentiste dans un orchestre à Cassel ${ }^{11}$, l'enseigne désormais chez des particuliers au début de la Seconde Restauration ${ }^{12}$. Au moral, les Souvenirs décrivent une Sophie à l'esprit vif, indépendant, volontiers espiègle ${ }^{13}$, « douce indulgente et toujours dévouée ${ }^{14}$ à l'égard de son aînée, très concernée par sa

\footnotetext{
6 Guest 1970 et 1974 ; Beaumont 1938 : 287-295; Olivesi 2015a.

7 Ravet $2003: 179-180$.

8 Taglioni $2017: 82-84$.

9 Ibid. : 613.

10 Ibid.

11 Michel $1934: 124$.

12 Taglioni $2017: 84$.

13 Ibid. : 74, 87-88, 123-124..

14 Ibid. : 60.
} 
formation et le développement de sa carrière ${ }^{15}$. C'est au contact de sa mère que Marie Taglioni donne libre cours à son goût pour la danse improvisée :

J'avais $[\ldots]$ l'habitude, lorsque ma mère jouait de la harpe de me mettre à danser et à mimer, mes inspirations venaient d'après le sentiment de la musique et ce sentiment m’est toujours resté ! Il m’a beaucoup aidée à varier ma danse ${ }^{16}$.

L'entre-soi féminin, que permettent les absences temporaires de Filippo, favorise son apprentissage d'une danse plus personnelle, hors des normes de la classe de danse.

Cette expérience d'une transmission matrilinéaire de la pratique des arts est minorée dans l'historiographie, la contribution de Sophie à la formation professionnelle de sa fille étant réduite à la confection de ses parures de scène ${ }^{17}$. On sait fort peu de choses en effet sur la biographie de Sophie Taglioni, mais elle semble avoir reçu une éducation artistique soignée et pluridisciplinaire. Elle pratique la harpe comme sa mère, Maria Sofia Stebnowska, chanteuse lyrique de formation qui se produit dans des concerts publics en qualité d'instrumentiste ${ }^{18}$. Avec sa sœur Élisabeth, elle s'exerce à la peinture et présente des toiles aux salons de l'Académie royale des arts de Suède entre 1802 et $1804^{19}$. Il n'est donc pas surprenant que Sophie Taglioni considère la carrière de Marie au théâtre comme un choix professionnel honorable. En effet, sa fille compose des petites comédies jouées en société20 ou danse en public à l'occasion de soirées regroupant leurs amis les plus proches:

Souvent des amies venaient passer la soirée chez nous, on ne manquait jamais de me faire danser, ce qui me faisait un grand plaisir, je m'habillais avec ma robe courte. Alors je composais quantité de pas, assurant que

15 Ibid. : 123.

16 Ibid. : 85.

17 Levinson 1929 ; Vaillat $1942: 20$; Winter $1974: 259$; Binney $1984: 108$.

18 Grut 2007 : 162. Son patronyme diffère selon les sources; «Stempkoska, Maria Sofia ", in Wilhelmina Stålberg \& Per Gustaf Berg (dir.), Anteckningar om svenska qvinnor, Stockholm, P.G. Berg, 1864-1866 : 354.

19 Nordin 2013: 17-18; «Karsten, Elisabeth Charlotta », in Wilhelmina Stålberg, Per Gustaf Berg, op. cit. : 214.

20 Taglioni $2017: 87$. 
mon professeur me les avait enseignés ; tous étaient ravis, émerveillés, je ne sais vraiment pas ce que j'exécutais devant eux, mais souvent je les faisais pleurer ${ }^{21}$.

Si dans ses Somvenirs Marie Taglioni s'autorise rétrospectivement l'énonciation du « grand plaisir» qu'elle ressentit, jeune fille, à danser devant un public, la protestation de modestie tempère sans tarder le récit de ce qui pourrait être interprété comme une coquetterie. Elle a intériorisé les normes de genre de son époque qui conduisent les jeunes filles à placer la composition sous le sceau du secret ou à adopter des conduites d'autocensure.

\section{La persistance des normes de genre}

Forge-t-elle sa connaissance empirique de la composition à la lecture des traités de codification de la danse ? ${ }^{22}$ Une chose est sûre: les traités de dramaturgie et de codification du ballet qui circulent jusqu'au Second Empire, toujours l'œuvre de professionnels masculins, ignorent ou minorent les pratiques d'improvisation et de composition féminines. La production normative de Jean-Georges Noverre et Carlo Blasis, qui fait référence au XIX ${ }^{e}$ siècle, efface la mémoire des activités compositionnelles de Marie Sallé, pourtant célèbre, que Noverre a bien connue de son vivant ${ }^{23}$, et n'en rapporte que les talents d'interprète ${ }^{24}$. Seule l'édition de 1807 des Lettres sur la danse de Noverre signale de rares cas d'improvisatrices qui ont pu inspirer Taglioni: Mlle Allard «composant elle-même ses entrées avec goût sans le recours des maitres, chose très-rare dans son

21 Taglioni : 85.

22 Veroli 2016 : 183, sur le contenu de sa bibliothèque en 1834.

23 McCleave 2007 a et $2007 \mathrm{~b}$.

24 Jean-Georges Noverre, Lettres sur la danse, et sur les ballets, Lyon, Aimé Delaroche, 1760 : 126-127, 164-165 ; Lettres sur la danse, et sur les ballets, Vienne, de Trattnern, 1767 : 116, 151-152 ; Lettres sur la danse et sur les ballets, Londres, Paris, Veuve Dessain junior, 1783 : 57, 97 ; Lettres sur la danse, sur les ballets et les arts, SaintPétersbourg, Jean Charles Schnoor, 1803, t. 1 : 63, 81, et 1804, t. $4: 77$; Lettres sur les arts imitateurs en général et sur la danse en particulier, Paris, Léopold Collin ; La Haye, Immerzeel et Cie, 1807, t. 1 : 293-294 et t. 2 : 103-104. 
sexe $»^{25}$ et Jacqueline Coulon qui « avait une intelligence rare, d'autant plus précieuse à l'Opéra qu'elle remplaçait avec facilité toutes les absences occasionnées par des indispositions feintes ou vraies $»^{26}$. Le pédagogue et maitre de ballet italien Carlo Blasis développe pour sa part une conception de la danse «impromptue ${ }^{27}$ comme exercice préalable faisant partie intégrante de la formation à la composition chorégraphique dans ses différents manuels publiés entre 1820 et 1830. Mais ses préconisations, loin d'être neutres, s'adressent exclusivement au «jeune danseur $»^{28}$ que le maitre de ballet aura reconnu «doué du génie de la composition et d'une imagination créatrice $»^{29}$.

Dans l'espace public, Marie Taglioni restreint sa pratique de la danse improvisée à des situations «limites». Lors de ses débuts à Vienne dans La Réception d'une jeune nymphe à la cour de Terpsichore ${ }^{30}$ le chef d'orchestre néglige d'effectuer une pause dans la musique :

[...] mon père le comprit tout de suite, aussi me criait-il de la coulisse de commencer, mais je n'entendais rien, je regardais toute cette salle remplie de monde $[\ldots]^{31}$.

Déroutée, elle n'a d'autre choix que d'improviser son entrée en scène :

$[\ldots]$ je me mis en position de commencer, mais je ne me retrouvais plus avec la musique, j'eus une sueur froide qui me parcourut tout le corps,

25 Jean-George Noverre, Lettres sur les arts imitateur en général et sur la danse en particulier, op. cit., t. 2 : 116.

26 Ibid. : 121.

27 Carlo Blasis, Manuel complet de la danse, comprenant la théorie, la pratique et l'bistoire de cet art depuis les temps les plus reculés jusqu'à nos jours, Paris, Roret, 1830 : 105.

28 Ibid.

29 Ibid. : 103 ; consigne présente dans son Traité élémentaire, théorique et pratique de l'art de la danse, Milan, Chez Joseph Beati et Antoine Tenesti, 1820:113; dans le Trattato elementare, teorico-pratico sull' arte del ballo, 1830, Forli, Tipografia Bordandini : 79 ; le The Code of Terpsichore, London, E. Bull, 1830 : 95, et le Code complet de la danse, Paris, Audin, $1830: 208$.

30 La Réception d'une jeune nymphe à la cour de Terpsichore, divertissement de Filippo Taglioni, création le 10 juin 1822 au Kärtnertortheater, Vienne.

31 Taglioni $2017: 95$. 
cependant je ne perdis pas la tête, et me mis à composer, quoi ? Je n'en sais rien, il faut croire que c'était bien, on applaudissait $[\ldots]^{32}$.

Et Filippo de la féliciter après le spectacle : «Tu aurais eu vingt ans de théâtre, que tu n'aurais pas pu avoir plus de présence d'esprit que tu n'en as montré ce soir. ${ }^{33}$ » Ici, son père valorise une compétence dans l'interprétation de la partition musicale placée au service de l'intérêt collectif (fluidité de la représentation théâtrale et préservation des recettes de l'entreprise) ; elle est d'autant plus admise que son invisibilité contribue au bon déroulement des ballets sans jamais mettre en péril la division sexuée du travail ni contester l'auctorialité du maître de ballet sur sa composition chorégraphique.

\section{Constructions de la légitimité artistique}

Intériorisant au fil de sa formation en danse que la revendication féminine de la composition constitue une transgression à la norme, Marie Taglioni trouve auprès de son père, qui l'accompagne dans sa carrière jusqu'en 1843, les ressources techniques et la bienveillance nécessaires à la singularisation de son style de danse : il l'aide à se forger « un genre à [elle] $»^{34}$ - une identité d'artiste-interprète ${ }^{35}$ - tout en l'incitant à conformer sa danse aux normes de genre de son époque. Filippo Taglioni interdit à sa fille tout geste ou attitude «qui manqu[e] de décence et de pudeur $»^{36}$ et établit durablement sa respectabilité grâce aux ballets qu'il compose à son intention. La technique de danse de Marie et les « filles de rêve » ${ }^{37}$ qu'elle interprète construisent un imaginaire séraphique que valorise la critique dramatique. Dès 1827, à Paris où Marie fait carrière jusqu'en 1837, le feuilletoniste du Constitutionnel note «dans les manières, dans les grâces, dans le talent de Mlle Taglioni, une sorte de pudeur et de

\footnotetext{
32 Ibid.

33 Ibid. : 96.

34 Ibid. : 93.

35 Olivesi 2017 : 205.

36 Louis Véron, Mémoires d'un bourgeois de Paris, Paris, G. de Gonet, 1854, t. 3 : 304 ; cité par Veroli $2016: 177$.

37 Corbin 2014.
} 
naïveté qui leur donnent un prix inexprimable ${ }^{38}$. Sa danse, "grâce naturelle et de bonne compagnie $»^{39}$, devient la représentation d'une morale sociale pudibonde ${ }^{40}$ qui remporte l'adhésion au sein de la plupart des organes de presse généralistes et spécialisés, toutes tendances politiques confondues. Son interprétation du personnage de la Bayadère dans l'opéra d'Auber inaugure son identification dans Le Globe à une "vierge chrétienne, chaste, voilée, humble et timide, dévouée, mélancolique ${ }^{41}$ que renforce ensuite la création des ballets de La Sylphide, La Fille du Danube ${ }^{42}$ (1836), les pas de l'Ombre et des Fleurs dansés à Paris en 1840 et 1844. Dans l'intervalle, Marie s'unit au comte Gilbert de Voisins, un fait qui contribue probablement à établir sa respectabilité dans le monde ${ }^{43}$. Enfin, la diffusion du motif de la sylphide, qui s'inspire clairement de l'iconographie mariale dans un premier XIXe siècle marqué par la promotion de la dévotion à la Vierge et la multiplication des congrégations féminines ${ }^{44}$, assoit durablement sa respectabilité auprès du public aristocratique et bourgeois de l'Opéra, en particulier féminin ${ }^{45}$. La singularité de sa danse, toujours comparée en 1860 au « rêve pudique d'une vierge » ${ }^{46}$, lui assure certes une forme de légitimité dans la composition du Papillon mais inscrit son assignation dans la «reproduction» du répertoire paternel :

Mlle Taglioni, la déesse de la danse, la sylphide, la grâce incarnée, celle qui ne ressemble à personne et à qui personne ne ressemble, Mme

38 «Nouvelles des théâtres », Le Constitutionnel, 13 août 1827 : 4.

39 «Théâtre de l'Opéra. Paresse. - Mlle Dorus. - Mlle Taglioni », Le Figaro, 11 janvier $1831: 3$.

40 Vielledent 2009 : 129-130.

41 «Académie royale de musique. Mademoiselle Taglioni », Le Globe, 7 octobre 1831, n²80, p. 1120, cité par Patrick Berthier dans Gautier 2007 : 126. Le Dieu et la Bayadère, ou la courtisane amoureuse, opéra en deux actes de François Auber sur un livret de Scribe, création le 13 octobre 1830 ; cf. Veroli 2016 : 177-178.

42 La Fille du Danube, ballet de Filippo Taglioni, livret d'Eugène Desmares, partition d'Adolphe Adam, création à l'Opéra le 21 septembre 1836.

43 Veroli $2016: 178$.

44 Langlois 1984 : 187-211; Joutard 1991 : 292.

45 Louis Véron, op. cit. : 304-305.

46 «Nouvelles », Les Coulisses, 4 novembre 1860 : n.p. 
Taglioni règle les pas et compose les tableaux. C'est pour la première fois qu'elle vient montrer aux autres ce qu'elle a admirablement exécuté ellemême. Nous allons la juger comme maitre de ballet, et nous sommes sûrs d'avance qu'elle saura trouver des effets inédits, qu'elle inventera de nouvelles combinaisons et que nous la reconnaitrons jusque dans le battement de l'aile des papillons : elle se connait en ailes !47

\section{Une vedette sous tutelle}

Car au fil des ans, les compositions chorégraphiques de Filippo sont devenues par contrat le "répertoire ${ }^{48}$ de Marie qui peut choisir ses rôles, se réserver l'exclusivité de danser certains pas, refuser d'en danser d'autres, demander le recrutement d'un maitre de ballet ou imposer le soliste masculin de son choix ${ }^{49}$. Outre qu'elle permet au père d'obtenir des emplois de maître de ballet là où la fille se produit, cette stratégie permet à Marie d'acquérir une autonomie dans sa pratique professionnelle. À cet égard, le contrat que Filippo lui négocie avec la direction de l'Opéra en 1831 est éclairant en ce qu'il stipule que Marie «ne pourra être obligée [...] à jouer des rôles ou danser des pas qui n'auraient pas été composés pour elle», qu'elle " pourra exiger que ses pas soient de la composition de son père »et, qu'à défaut, elle "aura la faculté de composer elle-même les pas qu'elle dansera $»^{50}$. La présence de son père, alors maitre de ballet à l'Opéra, rend peu probable l'exécution de cette clause, mais son énonciation montre qu'à 27 ans, Marie est reconnue compétente pour chorégraphier ses propres rôles. Cette clause témoigne en outre des relations de confiance nouées entre la fille et son père qui, par ailleurs, l'autorise à le remplacer lorsque sa santé l'empêche de donner des cours de danse de société à des jeunes filles de la bonne société

\footnotetext{
47 Henri Desroches, «La semaine », Le Constitutionnel, 26 août 1860 : n.p.

48 Bibliothèque-musée de l'Opéra (BMO), Fonds Taglioni, R 32, ms, $\mathrm{f}^{\circ} 1 \mathrm{r}^{\circ}$; R 42, $\mathrm{ms}, \mathrm{f}^{\circ} 1 \mathrm{r}^{\circ}$; R 46, $\mathrm{f}^{\circ} 1 \mathrm{r}^{\circ}$. Contrats de Marie Taglioni : Saint-Pétersbourg (1837); Kärntnertortheater, Vienne (1839); Milan (1840).

49 BMO, Fonds Taglioni, R 28, ms, $\mathrm{f}^{\circ} 1 \mathrm{v}^{\circ}$. Article 4, contrat de Marie Taglioni, King's Theatre, Londres, 1835.

50 Archives nationales (AN), AJ13 $129, \mathrm{~ms}, \mathrm{f}^{\circ} 1 \mathrm{v}^{\circ}$. Dossier Marie Taglioni, article 7 du contrat du 7 juin 1831.
} 
stuttgartoise - Marie note à ce propos combien « [sa] joie fut grande d'être acceptée $»^{51}$.

Dans ses Souvenirs, elle ne consigne aucune trace de désaccords qui ont pu l'opposer à son père, mais il est possible que sa tutelle pèse sur ses choix professionnels et retarde de plusieurs décennies son engagement assumé dans la composition chorégraphique. En 1833 déjà, le sujet la touche de près, ce que révèle une lettre adressée au marquis de Las Marismas où elle commente l'actualité théâtrale londonienne et jauge ses concurrentes avec force détails. L'évocation des sœurs Elssler qui vont bientôt se produire au King's Theatre interrompt brusquement son persiflage :

[...] il y a deux danseuses d'Allemagne qui doivent déjà y être, dans ce mois ce sont les demoiselles Esler (sic) on dit que la plus jeune est très jolie et danse très bien, il parait que si je puis l'emporter sur ce talent là, je n'ai plus aucune rivale à craindre. Je crois qu'elles viendront à Paris, l'aînée de ces deux dames monte les ballets elle-même ${ }^{52}$.

Banquier et bailleur de fonds de l'Opéra, le marquis est un proche de Louis Véron, alors directeur de l'Opéra, si bien que Marie, prudente, reste à sa place d'interprète et s'abstient d'exprimer une opinion personnelle.

\section{Taglioni, actrice de la féminisation du ballet}

Un quart de siècle plus tard, le tabou qui entourait l'investissement des femmes dans la composition chorégraphique et la pédagogie est levé. Si on considère les carrières des vedettes de la danse théâtrale à l'échelle européenne caractérisées par une forte mobilité géographique, Taglioni n'est pas au sens strict une pionnière ${ }^{53}$. À partir de 1830, des danseuses exercent, en plus de leur activité d'interprète, une fonction de chorégraphe de manière régulière et " par projet» au Her's Majesty Theatre ou au Drury Lane de Londres, au

51 Taglioni : 74. Les faits datent du séjour que les Taglioni réalisent à Stuttgart entre 1824 et 1828.

52 BMO, Fonds Taglioni, R. 67. Copie de lettre dactylographiée de Marie Taglioni à Alexandre-Marie Aguado marquis de Las Marismas, 14 mars 1833.

53 Garafola $1997: 7-8$. 
Théâtre Royal de Bruxelles, au Kärntnertortheater ou dans de nombreuses entreprises de spectacle italiennes - à cet égard, les trajectoires de Fanny Cerrito et Lucile Grahn sont paradigmatiques ${ }^{54}$. Certaines d'entre elles publient leurs propres livrets de ballet garantissant ainsi leur auctorialité sur les chorégraphies - une initiative que Marie Taglioni, auteure de plusieurs livrets ${ }^{55}$, ne mène pas à son terme. Dans certains cas, leur activité professionnelle est publiquement reconnue et elles sont intégrées dans la hiérarchie professionnelle de l'entreprise puisqu'elles sont titulaires d'un emploi.

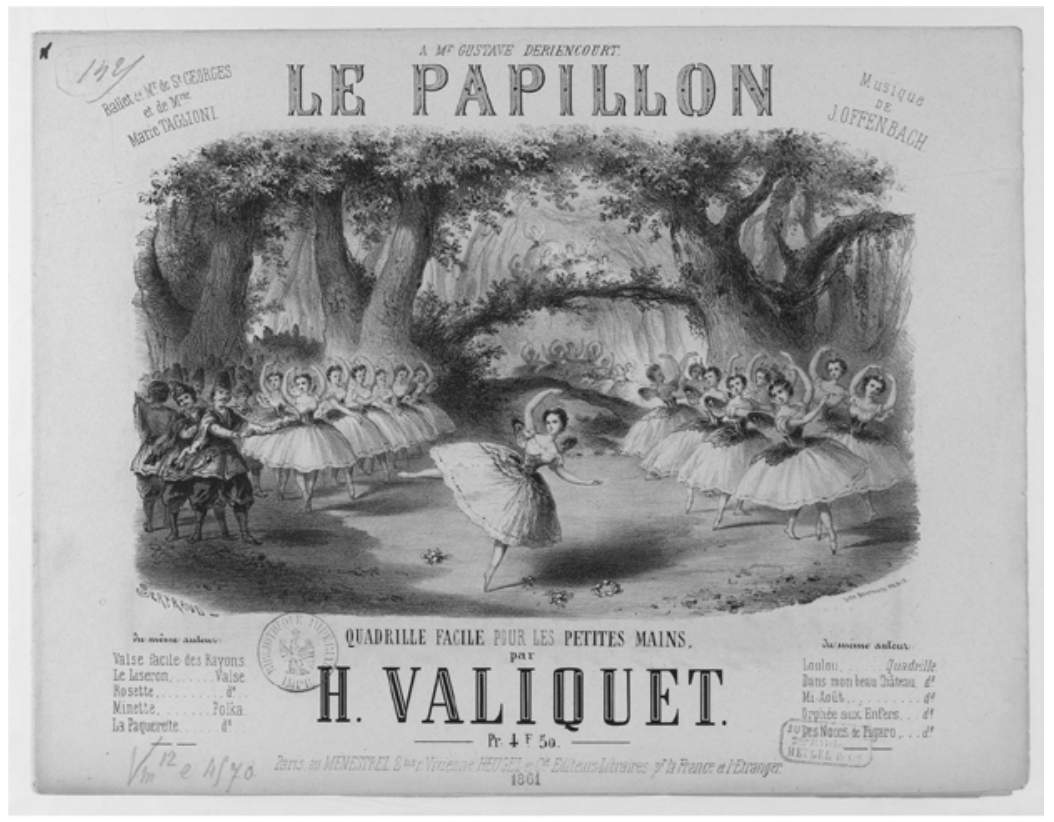

Fig. 2. Le Papillon, ballet composé Ballet en deux actes de Mme Marie Taglioni et de Mr de St. Georges. Musique de J. Offenbach... Quadrille facile (pour piano) pour les petites mains par H. Valiquet, Paris, Ménestrel, 1861 C BnF, Gallica.

54 Olivesi 2015a.

55 BMO, Fonds Taglioni, R 16, ms, n.d., 118 ff. Carnet de Marie Taglioni, livret de Pandore et projet de livret sans titre. 
Trois scènes londoniennes recrutent en effet des ballet mistresses : le Drury Lane déjà cité où œuvrent Miss Barnett dès $1831^{56}$ puis Madame Louise entre 1852 et 185457 , le Queen's Theatre et le City Theatre, scènes moins prestigieuses où Miss Rose et Madame Deulin sont actives au milieu des années $1840^{58}$. À la même époque, dans l'espace germanique, Joséphine Weiss est nommée balletmeisterin au théâtre Josephstadt de Vienne dès 183759 et la danoise Lucile Grahn exerce en cette qualité entre 1858 et 1875 à Leipzig puis au Munich Hoftheater ${ }^{60}$.

Bien qu'elle ne commente pas directement ces faits dans sa correspondance ni même dans ses Souvenirs, Marie Taglioni a sans nul doute observé cette dynamique de féminisation de la danse théâtrale. Aucune recherche ne l'a mesurée à l'échelle continentale, mais celle de l'Opéra peut être étudiée à l'aide des registres comptables qui recensent le personnel. Leur dépouillement systématique permet d'établir que cette féminisation du personnel artistique précède de loin la carrière de Taglioni ${ }^{61}$ : effectuée par le bas de la hiérarchie du corps de ballet durant la seconde moitié du XVIII ${ }^{\mathrm{e}}$ siècle, cette féminisation concerne à partir du Consulat les catégories d'emplois les plus qualifiées. Lorsque Marie est admise à l'Opéra, en 1827, les femmes représentent un peu plus de la moitié des effectifs du corps de ballet soit 51\%, et leur nombre continue de progresser pour atteindre $55 \%$ à son départ de l'Opéra en 1837 puis $60 \%$ des effectifs totaux lors de sa tournée d'adieu en $1844^{62}$. Au mitan du siècle, la part des femmes s'élève à $70 \%$ des effectifs du corps de ballet et dans la

56 «Dramatic Review », The Metropolitan, vol. II, December 1831 : 139; vol. III : February $1832: 53$; April 1832: 132 .

57 Richards $2015: 185$.

58 William Henry Oxberry, Oxberry's Budget of Plays, London, Vickers and Cleave, 1844, vol. $1: 198$.

59 "Weiss, Josephine», in Constant von Wurzbach, Biographisches Lexikon des Kaiserthums Österreich, Wien, Zamarski, t. 54, 1886 : 125-127.

60 Aschengreen 2013.

61 La Gorce 1979 ; Sadler 1987. AN : AJ13 1, AJ'13 15, AJ1316, AJ13 57, AJ13 190, $\mathrm{AJ}^{13} 230$; et $\mathrm{BMO}: \mathrm{AD} 68, \mathrm{PE}-1$ à PE-4.

62 BMO, Archives de l'Opéra. Personnel. États matrices, PE-2, ms, 1827, ff 280-284 ; PE3, ms, 1836-1837, ff 126-129 ; et PE-4, ms, 1844-1849, ff 5-8. 
catégorie des solistes, elles dominent largement avec 20 danseuses pour 9 danseurs ${ }^{63}$. En 1860-1861, lorsque Taglioni intègre l'Opéra en qualité de pédagogue, les danseuses solistes sont encore largement majoritaires ${ }^{64}$. Quelques années plus tôt, en 1853, la direction du théâtre avait confié à une ancienne danseuse, Mme Dominique, un poste de professeur de la classe élémentaire des filles ${ }^{65}$, ce qui donne à penser que l'entreprise de Marie Taglioni s'inscrit dans un contexte où la direction de l'Opéra et son ministère de tutelle ${ }^{66}$ s'ouvrent ponctuellement à l'examen de candidatures féminines.

\section{Un solide réseau professionnel}

On peut formuler quelque hypothèse sur la manière dont Taglioni a conçu sa candidature de pédagogue et de chorégraphe au cours de l'été 1858. Le croisement de sa correspondance avec le dépouillement des grands organes de presse parisiens montre qu'elle s'appuie sur un solide réseau politique et artistique au nombre desquels on peut citer Louis Véron devenu patron de presse influent, homme politique et fervent soutien de l'Empereur ${ }^{67}$; le comte de Morny, figure centrale du régime, protecteur d'Offenbach et artisan de la politique artistique de la capitale; Henri de Villemessant, directeur du Figaro qui chronique sans relâche l'actualité mondaine et professionnelle de Taglioni. Si elle cultive de longue date des liens de promotion respectifs avec Villemessant, fondateur en 1839 du journal élégant $L a$ Sylphide ${ }^{68}$, ses contacts avec le demi-frère de l'Empereur, plus récents, sont facilités par des mariages plaçant Taglioni dans son cercle familial élargi ${ }^{69}$.

63 Ibid., PE-4, ms, 1844-1849, f 69 ; AN, AJ ${ }^{13}$ 190, I.

64 Dawson $2006: 153$.

65 Guest 2014 [1953] : 7.

66 Yon $2012:$ 95-96.

67 Adolphe Robert, Gaston Cougny, Edgard Bourloton, Dictionnaire des parlementaires français, Paris, Bourloton, t. 5, 1891 : 508-509.

68 Henri de Villemessant, Mémoires d'un journaliste, Paris, E. Dentu, 1867 : 79.

69 Marie Taglioni entre dans le cercle de Morny au plus tard en 1852, cf. Arsène Houssaye, Les Confessions : souvenirs d'un demi-siècle, Paris, E. Dentu, 1885, t. 4 : 310 ; 
Une lettre que Louis Véron adresse à Marie Taglioni le 22 août 1858 indique qu'elle lui a confié ses projets - sans qu'on sache si le contenu de leurs échanges concerne la composition chorégraphique, un emploi de professeur ou les deux - mais la lettre atteste que Véron s'en est ouvert à Morny, concluant «Vous serez pour l'Opéra et pour les ballets une Providence! et tout ensuite viendra à point. Ce n'est pas à vous à demander, il faut qu'on vous prie et qu'on vous attire $»^{70}$. Véron patronne l'intégration de Taglioni au sein de l'Opéra, l'affichant à ses côtés dans sa loge lors de la représentation de La Sylphide le 17 novembre $1858^{71}$. Il organise avec le concours du directeur Alphonse Royer, un banquet en son honneur qui réunit l'élite du monde chorégraphique, musical et dramatique ${ }^{72}$. En même temps Marie Taglioni mobilise un réseau féminin qu'inaugure la comtesse Dash avec la publication dans Le Figaro, le 31 octobre, d'un portrait littéraire de l'ancienne danseuse en « bourgeoise $»^{73}$ accomplie :

Si on entrait chez elle sans se douter où l'on va, on se croirait chez une mère de famille bien rangée, bien obscure. On verrait un salon confortable, avec le piano classique, avec une table à ouvrage, sur laquelle sont déposés tous les instruments du travail féminin. Le métier à tapisserie est dans un coin, un fauteuil commencé par une main de fée y est attaché ${ }^{74}$.

Marie s'implique, au contraire, dans une intense vie mondaine qui lui permet de promouvoir la création du Papillon et d'asseoir ses activités de pédagogue au sein du Tout-Paris : réceptions et bonnes œuvres à l'instigation de $\mathrm{Mme} \mathrm{Scribe}^{75}$, épouse du dramaturge, se

sa fille Marie-Eugénie se marie en 1852 avec Alexandre Wassiljewitch Troubetzkoy, oncle de Sophie Troubetzkoy devenue comtesse de Morny en 1857.

70 BMO, Fonds Taglioni, ms, $\mathrm{f}^{\circ} 1 \mathrm{r}^{\circ}$. Lettre de Louis Véron à Marie Taglioni, Paris, 22 août [1858] ; cf. Vaillat $1942:$ 512-513.

71 Guest 2014 [1953] : 134.

72 «Petite chronique - Banquet Taglioni », Le Ménestrel, 5 décembre 1858 : 7.

73 Jacques Reynaud [comtesse Dash], «Portraits contemporains. XVIII. Marie Taglioni », Le Figaro, 31 octobre 1858 : 3. Taglioni avait recommandé la comtesse Dash à Eugène Scribe en 1847. Cf. Schopp 2014, sur les pseudonymes de la comtesse Dash.

74 Jacques Reynaud [comtesse Dash], op. cit.

75 Camille de Jailly [comtesse Dash], « Causerie », Le Figaro, 6 février 1859 : 2. 
succèdent, chez Rossini qui tient salon musical ${ }^{76}$ ou au domicile de Taglioni ${ }^{77}$, jusqu'à sa nomination officielle à l'emploi d'inspectrice des classes de danse en août 1860. Cette promotion répond à l'objectif de soumettre l'école à "des réformes ${ }^{78}$ auxquelles elle s'emploie en préconisant l'abolition de la mixité de l'enseignement, le renforcement de la formation dédiée aux jeunes filles et le recrutement de femmes pédagogues dans un rapport remis au ministre Achille Fould qui supervise alors l'administration de l'Opéra ${ }^{79}$. L'argument ne convainc pas le ministre selon lequel «jamais une femme n'a été appelée à exercer le Professorat d'une classe de Danse ${ }^{80}$, craignant en outre «que l'autorité de l'enseignement et de la discipline ne fut affaiblie entre les mains ${ }^{81}$ d'une pédagogue. Marie Taglioni doit sans doute sa carrière à l'intervention directe de Morny : Fould, qui pressait pourtant Royer d'ouvrir le recrutement aux maitres de ballet, change brutalement son fusil d'épaule en avril 1860 et la nomme au poste de professeur de la classe de perfectionnement ${ }^{82}$. On peut également déceler la main du comte à travers la présence remarquée de la famille impériale aux représentations du Papillon ${ }^{83}$; des marques de faveur que Taglioni et Offenbach cultivent en retour avec la publication d'une partition musicale dédiée à la comtesse de Morny ${ }^{84}$.

76 Camille de Jailly [comtesse Dash], « Causerie », Le Figaro, 6 mars $1859: 2$.

77 Guest 2014 [1953] : 135-136.

78 BMO, Fonds Taglioni, R 64, ms, f $1 \mathrm{r}^{\circ}$. Lettre d'Alphonse Royer à Marie Taglioni, Inspectrice, 12 août 1859.

$79 \mathrm{AN}, \mathrm{AJ}^{13}$ 479, ms. Note sur les classes de danse de l'Académie Impériale de Musique "par $\mathrm{M}^{\mathrm{me}}$ Taglioni ».

80 BMO, Archives de l'Opéra. Danse. Personnel. Dossiers personnels. Taglioni, Marie, 18591871, pièce 11, ms, fo $1 \mathrm{r}^{\circ}$. Lettre du ministre [A. Fould] à Alphonse Royer, 27 février 1860.

81 Ibid., f ${ }^{\circ} 1 \mathrm{v}^{\circ}$.

82 Ibid., pièce 8, ms, fo $1 \mathrm{r}^{\circ}$. Lettre d'Alphonse Royer au ministre, $1^{\text {er }}$ mars 1860.

83 Olivesi 2015b : 200-201.

84 Académie Impériale de Musique. Hommage à Madame la Ctesse de Morny, Le Papillon, Ballet de $M^{r}$ de St-Georges et de $M^{m e}$ Marie Taglioni, Musique de J. Offenbach, Le Papillon, Valse des Rayons, dansée par Mademlle Emma Livry, composée par Strauss, Paris, Au Ménestrel, Heugel et Cie, 1861, 9 p. 


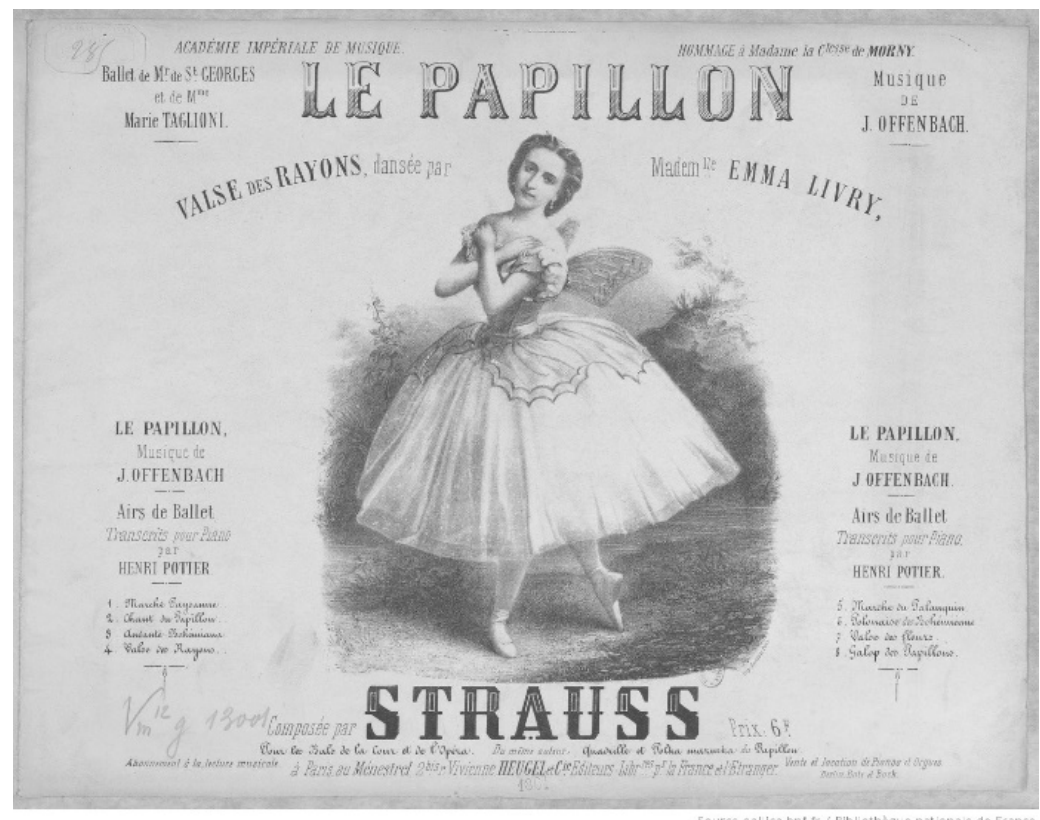

Fig. 3. Le Papillon, ballet composé. Ballet en deux actes de Mme Marie Taglioni et de Mr de St. Georges. Musique de J. Offenbach... Valse des rayons (pour piano)..., composée par Strauss, Paris, Heugel, 1861 C BnF, Gallica.

\section{Le prix du déclassement}

Marie Taglioni a su dépasser les censures et normes de genre de son époque. Avec persévérance, elle a mobilisé un capital symbolique et un réseau professionnel patiemment construits au fil de sa carrière d'interprète pour asseoir un début d'indépendance économique et favoriser l'expression de son désir - composer la danse. Son traitement, fixé à trois mille francs annuels en 1859, est revalorisé à six mille francs lors de son admission en qualité de professeur l'année suivante. La création du Papillon donne lieu à des manifestations de reconnaissance inédites à l'égard d'une femme : l'excellente réception critique de la chorégraphie fait écho aux ovations du public et légitime son statut de chorégraphe si bien que la direction de l'Opéra 
sollicite derechef ses compétences dans la composition ${ }^{85}$. Elle collabore aux chorégraphies du Carnaval des revues d'Offenbach aux Bouffes Parisiens ${ }^{86}$, mais sa stratégie de reconversion professionnelle atteint rapidement ses limites: le montant de ses droits d'auteur, aléatoire, ne peut compenser la modestie de son salaire qui équivaut à la moitié des émoluments perçus par ses concurrents maîtres de ballet. En fait, c'est au prix d'un double déclassement professionnel l'assignation à l'emploi de pédagogue et la spécialisation dans la danse de société - que Taglioni peut temporairement exercer ses compétences de chorégraphe. Si la féminisation du corps de ballet de l'Opéra, la puissance de son réseau relationnel et sa respectabilité sont des facteurs qui favorisent son intégration dans l'entreprise de spectacle, l'activité compositionnelle engage l'exercice d'un pouvoir de direction sur le personnel artistique qu'elle exerce certes dans les faits lors des répétitions du Papillon et de Zara mais dont la légitimité lui est refusée. Contrainte de diversifier ses activités professionnelles, elle n'a d'autre choix que de s'engager dans des activités jugées plus conformes à son sexe : l'enseignement aux amateurs dans la bonne société parisienne et l'invention des « danses de ville ${ }^{87}$.

\section{Bibliographie}

AschengreEn Erik, 2013, "Grahn, Lucile», in Béatrice DiDIER, Antoinette Fouque \& Mireille Calle-Gruber (dir.), Dictionnaire universel des créatrices, Paris, Des femmes, Antoinette Fouque, vol. 2, p. 1806.

Beaumont Cyril W., 1938, Complete Book of Ballets, A guide to the principal ballets of the nineteenth and twentieth centuries, New York, Grosset \& Dunlap, G.P. Putman's sons.

BINNEY Edwin 3 $3^{\text {rd }}$ 1984, "Longing for the ideal: images of Marie Taglioni in the Romantic Ballet », Harvard Library Bulletin, XXXII, p. 105-148.

CORBIN Alain, 2014, Les Filles de rêve, Paris, Fayard.

85 AN, AJ13 499, I, 2 ff. Lettre de Marie Taglioni à Émile Perrin, n.d. [ca 1862-1870].

86 «Revue de Carnaval avec prologue. - Mardi Gras », Le Ménestrel, 12 février 1860 : 86.

87 Paul d'Ivoi, «Le bal de l'hôtel d'Albe », Le Figaro, 29 avril 1860 : 1 ; La Taglioni. Nouvelle danse de salon réglée par Mme Marie Taglioni, musique de Pbilippe Stutr, Paris, Heugel, Au Ménestrel, 1863, pag. mult. 
DAwSON Jennifer A., 2006, «Danseuses as working women: ballet and female waged labor at the Paris Opéra, 1830-1860», Ph.D., Philosophy in Dance History and Theory, sous la dir. Linda J. Tomko, University of California Riverside.

GARAFOLA Lynn (ed.), 1997, Rethinking the Sylph: new perspectives on the romantic ballet, Hanover, London, Wesleyan University Press, 1997.

—, 2005, "Where are ballet's women choreographers? ", in Lynn GARAFOLA, Legacies of twentieth century dance, Middletown, Wesleyan University Press, p. 215-228.

Gautier Théophile, 2007, Euvres complètes. Section VI, Critique théatrale, Tome 1, texte établi, présenté et annoté par Patrick Berthier, avec la collaboration de François Brunet, Paris, H. Champion.

GruT Marina, 2007, Royal Swedish Ballet: history from 1592 to 1962, Hildesheim, G. Olms.

Guest Ivor, 1970, Fanny Elssler, London, Adam \& Charles Black.

—, 1974, Fanny Cerrito, the life of a romantic ballerina, London, Dance Books.

—, 2014 [1 ${ }^{\text {st }}$ ed. London, Adam and Charles Black, 1953], The Ballet of the Second Empire, 1858-1870, Binsted, Dance Books.

Gustche-Miller Sarah, 2015, Parisian Music-Hall Ballet, 1871-1913, Rochester, University of Rochester Press, Eastman Studies in Music.

LA GORCE Jérôme de, 1979, «L’Académie Royale de Musique en 1704, d'après des documents inédits conservés dans les archives notariales ", Revue de musicologie, 65/2, p. 160-191.

LANGLOIS Claude, 1984, Le Catholicisme au féminin. Les Congrégations françaises à supérieure générale au XIXe siècle, Paris, Éditions du Cerf.

JOUTARD Philippe (dir.), 1991, Du roi Très Chrétien à la lä̈cité républicaine (XVIII -XIXe siècle), tome 3 de Jacques LE GOFF \& René RÉMOND (dir.), Histoire de la France religieuse, Paris, Seuil, coll. «L'Univers historique ».

Levinson André, 1929, Marie Taglioni, 1804-1884, Paris, F. Alcan.

MARQuiÉ Hélène, 2008, «'On peut s'étonner de son succès vraiment considérable et persistant'», Résonances-Femmes, 10 : «La Reconnaissance » (dir. Claude CoHENSAFIR), p. 107-124.

—, 2013, « Entrez dans la danse, créez de la danse ! - Maîtresses de ballet à la Belle Époque », in François Le GuenNEC \& Nicolas-Henri Zmelty (dir.), La Belle Époque des femmes? 1889-1914, Paris, L'Harmattan, p. 75-88.

_, 2015, «Enquête en cours sur Madame Stichel (1856-ap. 1933). Quelques pistes de réflexion ", Recherches en danse, 3: "Perspectives genrées sur les femmes dans l'histoire de la danse » (dir. Hélène Marquié \& Marina Nordera) [en ligne : http://danse.revues.org/974]. 
—, 2016, Non, la danse n'est pas un truc de filles! Essai sur le genre en danse, Toulouse, Éditions de l'Attribut.

MCClEAve Sarah, 2007a, "Marie Sallé, a wise professional woman of influence », in Lynn Matluck Brooks (ed.), Women's Work: making dance in Europe before 1800, Madison, University of Wisconsin Press, p. 160-182.

—, 2007b, "Marie Sallé and the Development of the Ballet en action », Journal of the Society for Musicology in Ireland, 3-8, p. 1-23.

Michel Artur, 1934 [15 octobre], «Les ballets de Philippe Taglioni», Archives internationales de la danse, 4, p. 122-125.

NorDIN Jonas, 2013, «Handtechningar från tre sekler: Kepe-samlingen i Kungliga biblioteket », Biblis, 62, p. 2-24.

Olivesi Vannina, 2015a, «Fanny Cerrito chorégraphe. La réception de Gemma dans la presse parisienne (mai-juin 1854)», Recherches en danse, 3 : «Perspectives genrées sur les femmes dans l'histoire de la danse» (dir. Hélène MARQuiÉ \& Marina NORDERA) [en ligne http://danse.revues.org/896].

—, 2015b, «Entre création et transmission. La réception du Papillon de Marie Taglioni en 1860 », in Jacques Guilhaumou, Karine Lambert \& Anne Montenach (dir.), Genre, Révolution, Transgression. Études offertes à Martine Lapied, Aix-en-Provence, Presses universitaires de Provence, coll. « Penser le genre », p. 195-203.

—, 2017, «Le vedettariat dans le ballet romantique : l'exemple de Marie Taglioni à l'Opéra (1827-1838) », in Florence FILIPPI, Sara Harvey \& Sophie Marchand (dir.), Le Sacre de l'acteur. Émergence du vedettariat théatral de Molière à Sarah Bernhardt, Paris, Armand Colin, coll. «U », p. 203-211.

RAVET Hyacinthe, 2003, «Professionnalisation féminine et féminisation d'une profession : les artistes interprètes de musique », Travail, genre et sociétés, I, 9, p. 173-195.

Richards Jeffrey, 2015, The Golden Age of Pantomime: Slapsticks, Spectacle and Subversion in Victorian England, London, New York, I.B. Tauris.

SADLER Graham, 1987, "The Paris Opéra dancers in Rameau's day: a little-known inventory of 1738 ", in Jérôme DE LA GORCE (dir.), Jean-Philippe Rameau, actes du colloque international (Dijon, 1983), Paris, Champion ; Genève, Slatkine, p. 519-531.

SCHOpp Claude, 2014, «Sous le manteau d'Alexandre Dumas, ou les douze noms de la comtesse Dash », L'Ull Critic, Segona Etapa, revista d'estudis de llenga i literatura franceses $i$ francofones, 17-18: «Les romancières sentimentales : nouvelles approches, nouvelles perspectives » (dir. Àngels Santa \& Maria Carme Figuerola), p. 93-115.

TAGLIONI Marie, 2017, Souvenirs. Le manuscrit inédit de la grande danseuse romantique; édition établie, présentée et annotée par Bruno Ligore; préface de Flavia Pappacena, avec un essai d'Audrey Gay-Mazuel, Saint-Denis-sur-Sarthon, Gremese, Éditions de Grenelle. 
Vaillat Léandre, 1942, La Taglioni, ou la vie d'une danseuse, Paris, Albin Michel.

Veroli Patrizia, 2016, «Taglioni-mania. Les images de Marie Taglioni dans la culture visuelle de son époque ", in Madison U. Sowell, Debra H. Sowell, Francesca Falcone \& Patricia Veroli, Icônes du ballet romantique. Marie Taglioni et sa famille, Rome, Gremese, p. 169-183.

Vielledent Sylvie, 2009, "La réception de La Sylphide », Bulletin de la Société Théophile Gautier, 31 : "Gautier et les arts de la danse », p. 121-134.

Winter Marian H., 1974, The Pre-Romantic Ballet, London, Pitman.

Yon Jean-Claude, 2012, Une Histoire du théatre à Paris de la Révolution à la Grande Guerre, Paris, Flammarion.

Le portrait ci-après fait partie d'une série de photographies réalisées dans le studio de Disdéri en 1858. La pose de Marie Taglioni, l'air rêveur, main gauche s'appuyant sur une pile de livres, est un motif convenu des cartes de visite du Second Empire. Elle revêt cependant un sens équivoque à une époque où la presse disqualifie les femmes chorégraphes en les désignant de «bas-bleu chorégraphique ». On ne peut exclure l'hypothèse que la chorégraphe tente ici d'affirmer discrètement son statut professionnel, à l'image du maître de ballet Arthur Saint-Léon qui se fait représenter auprès d'une console jonchée de livres, de manuscrits et de partitions. Quatre ans plus tard, lorsque La Galerie des Contemporains consacre à la chorégraphe une notice biographique qui euphémise sa contribution au Papillon pour valoriser sa fonction pédagogique et protectrice à l'égard d'Emma Livry, les éditeurs illustrent la notice d'une photographie plus formelle où Taglioni, le regard fermement dirigé vers l'objectif, replie son avant-bras sur son buste dans une attitude clairement distanciée par rapport aux livres. 


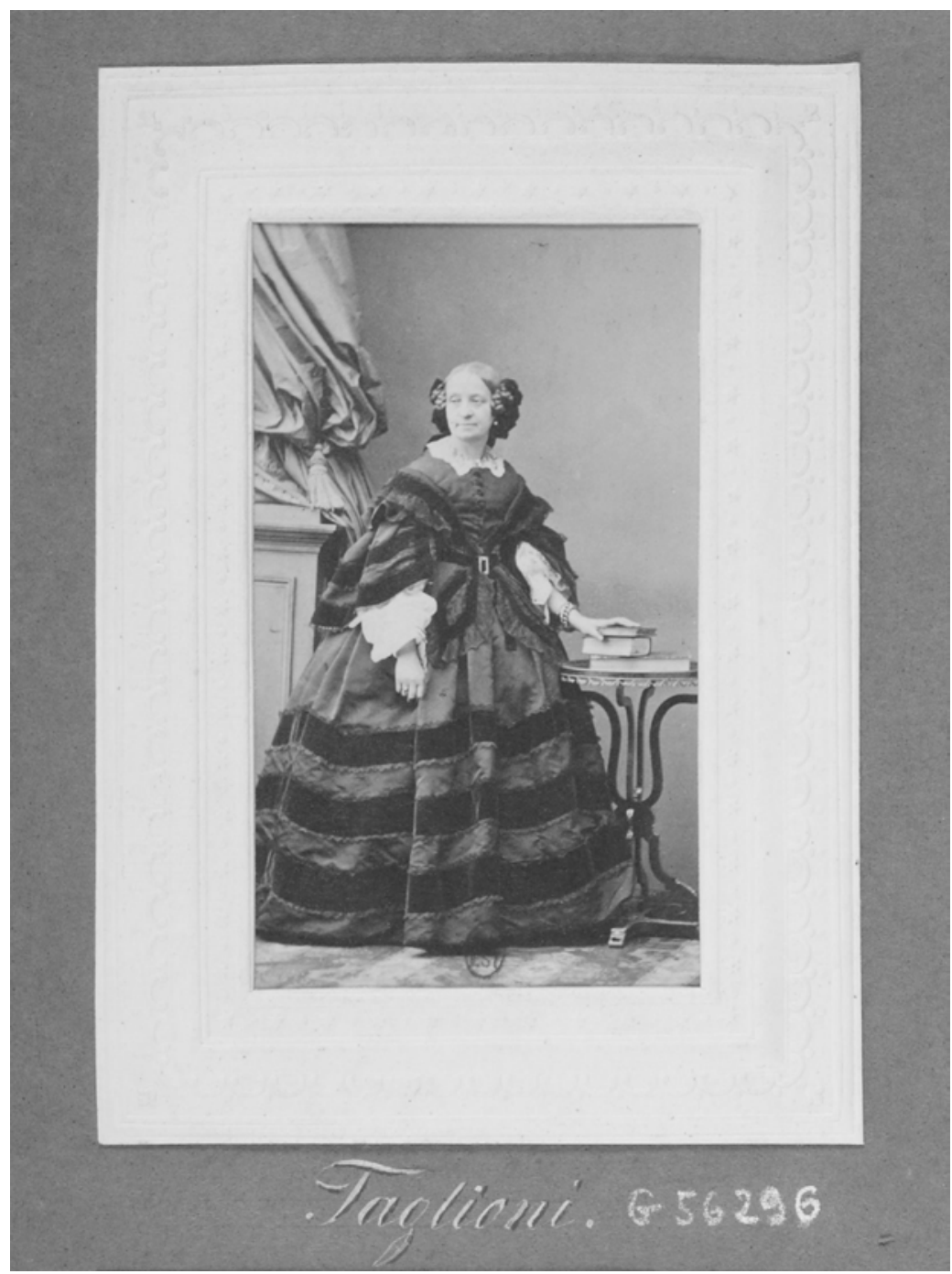




\section{NOTE A L'ATTENTION DE CHARLES RUELLE POUR DROITS ILL.}

J'ai eu la réponse de la $\mathrm{BnF}$ par mail : on peut directement télécharger les images sur Gallica et ne pas payer, si la définition des images convient à Clio. Par contre, si Clio veut des images en haute définition, il faut payer 25 euros par image. Personnellement, j'ai l'impression que les images de Gallica suffisent et que ce n'est pas nécessaire de payer.

Par contre, il faut régler cette fameuse redevance. J'ai cherché sur internet le $\mathrm{n}^{\circ}$ de TVA de Clio mais je n'ai pas trouvé (je n'ai pas pensé à saisir celui de Belin).

Voici le lien vers le formulaire pour le règlement de la redevance, pour que tu voies les informations à saisir :

https://espacepersonnel.bnf.fr/views/vel/declaration utilisation commerciale.jsf?pr ovenance $=$ null

Pour finir et faciliter les échanges, voici la liste des 3 images (lien vers Gallica) :

\section{M.me Taglioni / Disdéri phot. $\mid$ Gallica}

Le Papillon, ballet... , musique de J. Offenbach, . Valse des rayons (pour piano)... \begin{tabular}{l|l|l} 
composée par Strauss,... & Gallica \\
\hline
\end{tabular}

Le Papillon, ballet... , musique de J. Offenbach, . Quadrille facile (pour piano) pour les petites mains par $\mathrm{H}$. Valiquet $\mid$ Gallica

Voici le lien vers le document BnF où se trouve la photographie de Taglioni posant la main sur les livres (en haut à gauche de l'image) :

http://gallica.bnf.fr/ark:/12148/btv1b8438660q/f9.item

(Ici la notice biblio : http://catalogue.bnf.fr/ark:/12148/cb423232348)

Un portrait presque identique, mais en moins bon état, se trouve dans les fonds de la Cité de la musique (s'il s'avère que la BnF demande des droits élevés, cette information peut vous être utile) :

http://collectionsdumusee.philharmoniedeparis.fr/doc/MUSEE/0161541 